\title{
Labyrinthe
}

32 | 2009 (1)

Le petit théâtre intellectuel

\section{Deux « coups de théâtre »}

Le refus par Sartre du prix Nobel (octobre 1964), suivi de l'annulation de ses conférences à Cornell University (mars 1965)

\section{Laurent Ferri}

\section{(2) OpenEdition}

12 Journals

Édition électronique

URL : http://journals.openedition.org/labyrinthe/4009

DOI : $10.4000 /$ labyrinthe.4009

ISSN : 1950-6031

Éditeur

Hermann

Édition imprimée

Date de publication : 19 juin 2009

Pagination : 135-146

ISBN : 978-2-7056-6885-3

Référence électronique

Laurent Ferri, « Deux « coups de théâtre » », Labyrinthe [En ligne], 32 | 2009 (1), mis en ligne le 01

février 2011, consulté le 30 avril 2019. URL : http://journals.openedition.org/labyrinthe/4009 ; DOI :

$10.4000 /$ labyrinthe.4009

Propriété intellectuelle 


\section{DEUX «COUPS DE THÉÂTRE » Le refus par Sartre du prix Nobel (octobre 1964), suivi de l'annulation de ses conférences à Cornell University (mars 1965)}

Le récit du refus par Sartre du prix Nobel et de l'annulation de ses conférences à Cornell University trouve aisément sa place dans un numéro sur le « petit théâtre intellectuel ». Les coups d'éclat de Sartre, outre qu'ils témoignent d'un goût et d'une maîtrise de la mise en scène, pourraient fournir la trame de saynètes tragi-comiques sur l'engagement intellectuel pendant la guerre froide. Comme je n'ai pas ce talent, je me contenterai de mettre à la disposition des lecteurs de Labyrinthe les éléments du dossier, tel que nous pouvons le reconstituer à partir de biographies publiées $^{1}$ et d'archives inédites conservées au sein de la bibliothèque de Cornell, une des dix ou quinze plus importantes universités du monde, située à cinq heures de route à l'ouest de New York.

Notre hypothèse serait la suivante: comme me l'a fait remarquer Annie Cohen-Solal - qui travaille depuis longtemps sur le thème " Sartre et les États-Unis »- les deux refus obéissaient initialement à des logiques bien différentes. Toutefois, je m'autorise à penser que la proximité dans le temps, la volonté constante de cohérence de la part de Sartre, enfin la théâtralité des situations invitent à les rapprocher dans un même article. Alors que le philosophe engagé avait bien fait comprendre au jury Nobel, dès le 14 octobre 1964, qu'il n'accepterait pas le prix au nom du refus des honneurs et de la « récupération », il choisit de donner à son geste une justification nettement « anti-américaine » lorsque la récompense lui fut décernée tout de même (le 22 octobre); le deuxième coup de théâtre, passé largement inaperçu en France mais qui devait avoir des conséquen-

\footnotetext{
1. En particulier, pour ce qui est du Nobel, la synthèse publiée par Michel Contat dans Quai Voltaire, 6, 1992, et Pour Sartre, Paris, Presses Universitaires de France, 2008. Alors que l'affaire du Nobel a suscité de très abondants commentaires, l'épisode Cornell, qualifié bizarrement d' " incident mineur » par Denis Bertholet dans sa biographie (Sartre, Paris, Plon, 2000, p. 473) n'a pas, à ma connaissance, occasionné d'étude approfondie.
} 
ces durables pour la réception de Sartre aux États-Unis, survint lorsqu'il annula une série de conférences prévues pour le centième anniversaire de Cornell University, au lendemain des bombardements sur le NordVietnam (février-mars 1965).

Le prestige international de Sartre était immense, dans les années 1960. Il fut encore rehaussé par la publication en avril 1964 des Mots, un essai de virtuosité autobiographique commencé onze ans (!) plus tôt, et par lequel il semblait «scelle [r] ses adieux à la littérature [par] un patient travail d'artisan, qu'il ne consacra à aucune de ses œuvres ${ }^{1} »$. C'est à ce moment que l'Académie Nobel commença à les considérer. C'est aussi à ce moment que Cornell University, où la francophilie était à son apogée ${ }^{2}$, prit l'initiative d'inviter Jean-Paul Sartre dans le cadre des prestigieuses «Conférences Messenger sur les progrès de la civilisation » (Messenger Lectures on the Evolution of Civilization). $\mathrm{La}$ cheville ouvrière du projet était un certain Jean-Jacques Demorest, à la fois Chair des études littéraires romanes, mais aussi, et de manière plus inattendue, conseiller technique au cabinet de Christian Fouchet, ministre de l'Éducation nationale du Général de Gaulle. On sait l'estime relative que ce dernier pour le philosophe engagé: en septembre 1960, il aurait dit à son ministre de l'Intérieur, au moment du Manifeste des 121 sous-titré « Déclaration sur le droit à l'insoumission dans la guerre d'Algérie »:

\footnotetext{
1. Annie Cohen-Solal, Sartre, un penseur pour le Xxi siècle, Paris, Gallimard, 2005, p. 108.

2. Cornell University (Ithaca, New York) fut fondée en 1865 par un duo improbable: Ezra Cornell, élevé par les Quakers et milliardaire du télégraphe, et l'historien, diplomate, voyageur, bibliophile et militant anti-religieux Andrew Dickson White. Cornell eut toujours dans les milieux conservateurs américains une réputation sulfureuse d'université libérale et athée (Godless). Le modèle français de laïcité et de stricte séparation de l'Église et de l'État y fit certainement beaucoup pour l'épanouissement de la francophilie dans les années 1870-1914. Le deuxième âge d'or fut la conséquence du regain obscurantiste aux États-Unis (qui contrastait avec la grande liberté intellectuelle et artistique en France, comme le prouva en 1959 la publication à Paris de Lolita - Vladimir Nabokov était professeur de littérature à Cornell depuis dix ans), et de la politique gaulliste d'indépendance vis-à-vis des Américains. En novembre 1963, le président du Cornell Board of Trustees (ou conseil d'administration), l'avocat d'affaires et diplomate Arthur Dean, dont on dit qu'il persuada le président Johnson d'arrêter les bombardements sur le Nord-Vietnam en 1968, avait acheté pour Cornell Library l'essentiel des archives privées de La Fayette (Collection Fabius) - avec la bénédiction d'André Malraux, ministre d'État chargé des Affaires Culturelles. Malraux voyait dans les trésors du patrimoine national les meilleurs ambassadeurs de la France : on sait comment, lors de la visite à Paris du couple présidentiel américain en mai 1962, il avait glissé à l'oreille de Jacqueline Kennedy: « Je vais vous envoyer la Joconde », et comment il tint parole - Mona Lisa fut exposée à la National Gallery de Washington de l'automne 1962 au printemps 1963, sous la protection de militaires en grand uniforme. Margaret Leslie Davis, « Mona Lisa in Camelot », Vanity Fair, novembre 2008.
} 
« On ne met pas Voltaire en prison. » Je partage ici l'avis du Sartrologue Michel Contat: on ne peut vraiment comprendre les " coups de théâtre » de Sartre sans les rapprocher de la « théâtralité » de la politique gaullienne (la prise du pouvoir en 1958, « Je vous ai compris », «Vive le Québec libre », la fuite à Baden-Baden, etc.). « Le principal opposant à Charles de Gaulle, le seul qui soit internationalement célèbre, Jean-Paul Sartre, est poussé à théâtraliser son opposition par le fait même qu'il a affaire à un comédien sur la scène politique ${ }^{1}$. »

Le 14 août, Demorest écrivit au président James A. Perkins la lettre suivante, sur papier à en-tête du « Cabinet du Ministre de l'Éducation nationale ». Il savait que Perkins attachait beaucoup d'importance aux Messenger lectures, même si, en avril 1964, il n'avait pu, comme le voulait la tradition, souhaiter la bienvenue au speaker ... car il assistait à une réunion au ministère de la Défense (Pentagon - Advisory Commitee on Education in the Armed Forces) ${ }^{2}$. La prétention et le conformisme social de l'universitaire-technocrate rendent son style particulièrement savoureux:

Cher Président Perkins,

M. Jean-Paul Sartre vient de me donner son accord au sujet de la série de conférences Messenger: il sera notre invité au printemps 1965. Loin de se montrer difficile ou capricieux, Sartre m'est apparu comme un homme simple et courtois [...]. Je vous écris directement pour vous faire part de quelques libertés que je me suis autorisé à prendre lors des négociations préliminaires - il me paraît normal que vous en soyez informé puisque l'invitation officielle sera signée par vous. 1) j'ai bien insisté dès le départ sur le fait qu'à Cornell, qui fut historiquement la première université sans affiliation religieuse, l'esprit critique s'épanouit librement. Je pensais que cet argument porterait, et ce fut le cas. 2) Le comité de sélection s'étant mis d'accord sur le fait qu'il est encore préférable d'avoir un orateur s'exprimant en français plutôt que dans un anglais très approximatif (broken English), j'ai cru bon de suggérer un sujet littéraire plutôt que purement philosophique, qui rendrait les conférences encore plus difficiles à suivre dans une langue étrangère:

\footnotetext{
1. Pour Sartre, p. 551.

2. Les universités furent mises à contribution dans la guerre froide. Dans une institution comme Cornell, où l'entraînement militaire avait été obligatoire jusqu'en 1956, le président devait tenir compte de la situation géopolitique dans l'organisation des programmes et la répartition des ressources.
} 
j'ai proposé "Flaubert » - qui offre par surcroît l'avantage de ne pas occasionner a priori de "sorties » sur Ben Bella, Castro, ou Mao ${ }^{1} . . .3$ 3) J'ai fixé le nombre de conférences à cinq... 4) J'ai bien précisé que Cornell lui enverrait assez longtemps à l'avance son billet d'avion allerretour Paris-Ithaca, classe "économie ", et qu'il serait logé aux frais de l'Université. Toutefois, je sentais qu'il hésitait encore à nous donner son accord définitif, tant que l'étonnante - et beaucoup trop intelligente - Madame de Beauvoir n'était pas invitée elle aussi. Je pris sur moi de foncer-mais avec tact-et je lui dis que «bien sûr, Cornell serait ravie de recevoir également Mme de Beauvoir, si elle se souhaite se joindre à vous » [...] Je me dois de vous expliquer que Sartre et Beauvoir vivent ensemble, pour ainsi dire maritalement, depuis vingt-cinq ans ${ }^{2}$. Leurs détracteurs insinuent d'ailleurs qu'ils sont secrètement mariés, mais qu'ils n'en laissent rien paraître du fait de leur morale "émancipée ». Bref, si Mme de Beauvoir accompagne M. Sartre, il me semble qu'il faudrait prévoir deux chambres contiguës [connecting rooms]. Pardonnez-moi d'entrer ainsi dans les détails: mais Sartre étant, selon toute probabilité, la personnalité la plus éminente que Cornell soit appelée à recevoir dans les années à venir, j'ai pensé que vous souhaiteriez disposer de tous les éléments nécessaires à une organisation sans faille ${ }^{3}$.

C'est donc sous le signe de la soigneuse séparation des genres que Sartre était invité par l'habile Desmorest: genres littéraires (on parlerait de Flaubert, mais si possible pas d'événements politiques récents); genres sexuels ou genders, puisque Sartre et Beauvoir feraient si possible chambre séparée.

Le 7 mai 1964, Perkins signa l'invitation à Sartre. La lettre suggérait comme sujet « Flaubert - un sujet qui, j'en suis sûr, serait du plus haut intérêt pour notre communauté, et pour les nombreux petits collèges de la région ", et indiquait le montant très modeste de la rémunération, 2000 dollars $^{4}$ pour cinq conférences prononcées entre le 5 et le 14 avril

\footnotetext{
1. Il est significatif que Desmorest, fonctionnaire gaulliste, n'exprime aucune inquiétude quant à la possibilité que Sartre aborde la question du rôle des États-Unis en Asie du Sud-Est.

2. La liaison entre Sartre et Beauvoir avait en fait commencé en 1929.

3. Cornell University, Perkins Papers, \# 3-10-1022, box 6, folder 2. Je traduis.

4. À l'époque, n'importe quel professeur réputé se voyait offrir en moyenne 400 dollars pour une conférence.
} 
1965. Il n'était fait aucune mention de Beauvoir, Perkins se contentant de préciser que «le logement consistera en deux chambres contiguës »...

Cette invitation tombait-elle mal? Au moins depuis l'exécution d'Ethel et Julius Rosenberg, et la publication de l'article « Les animaux malades de la rage » (dans Libération, le 22 juin 1953), Sartre avait l'anti-américanisme chevillé au corps. Comme cette notion d'anti-américanisme fait l'objet d'usages divers et contestables, on devrait dire qu'il montrait une hostilité sans nuances au «système américain », idéologie dominante, institutions, et mode de vie des classes moyennes confondus (il semble que Sartre avait la même aversion pour les comics et Walt Disney, le fédéralisme tempéré, les études payantes, ou Wall Street).

Lorsque les premières rumeurs lui parvinrent, fin septembre, de l'attribution probable du prix Nobel, « l'équipe des Temps modernes, au grand complet, [s'exprima] en faveur de l'acceptation. Mais leur approbation ne [tenait]-elle pas à leur âge (la quarantaine) et à l'impact d'un Nobel pour la revue? Simone de Beauvoir [chercha] l'avis de gens plus jeunes et plus radicaux. Ils réagirent violemment. Accepter serait « un enterrement de première classe, une annexion au monde bourgeois ». La raison de cette colère [était] bien compréhensible: il y a quelques années, le jury du prix Nobel [avait] attribué le prix à l'auteur d'un méchant roman, Boris Pasternak, « pour embarrasser l'URSS. » Dont acte. On ne [pactiserait] pas avec ces gens ${ }^{1} »$. Certes, la lettre en forme de refus par avance que Sartre adressa au secrétaire du Comité Nobel le 14 octobre ne développait pas cet argument, mais revenait sur le thème du double refus du regard des autres et des honneurs - c'est-à-dire que Sartre refusait d'être $v u$ comme un grand écrivain recherchant les honneurs :

\section{Monsieur le Secrétaire,}

D'après certaines informations dontj'ai eu connaissance aujourd'hui, j'aurais, cette année, quelques chances d'obtenir le prix Nobel. Bien qu'il soit présomptueux de décider d'un vote avant qu'il ait eu lieu, je prends à l'instant la liberté de vous écrire pour dissiper ou éviter un malentendu.

Je vous assure d'abord, Monsieur le Secrétaire, de ma profonde estime pour l'Académie suédoise et pour le prix dont elle a honoré tant d'écrivains. Toutefois, pour des raisons qui me sont personnelles et pour

1. Denis Bertholet, Sartre..., p. 467. 
d'autres, plus objectives, il n'y a pas lieu de développer ici, je désire ne pas figurer sur la liste des lauréats possibles et ne peux ni ne veux-ni en 1964 ni plus tard-accepter cette distinction honorifique.

Je vous prie, Monsieur le Secrétaire, d'accepter mes excuses et de croire à ma très haute considération ${ }^{1}$.

Cependant, après que le Prix lui fut décerné tout de même, Sartre décida de se saisir de l'occasion pour réaffirmer de fortes convictions éthiques et politiques. Son texte fut déposé par Carl-Gustav Bjurström, son traducteur en suédois, auprès de l'Ambassade de Suède à Paris; il fut ensuite diffusé par l'Agence France-Presse, et parut le 24 octobre en suédois dans le Dagens Nyheter, et en français («traduit du suédois ») dans Le Monde, sous le titre: « L'écrivain doit refuser de se laisser transformer en institution ». Avec un luxe d'explications exagéré, et dans une langue de bois assez pénible, Sartre affirmait tout d'abord que son refus s'inscrivait dans le droit fil de l'attitude qu'il avait toujours eue vis-à-vis des honneurs officiels (il avait refusé la croix de la Légion d'Honneur en 1945 et n'avait jamais désiré entrer au Collège de France « comme me l'ont suggéré quelques-uns de mes amis »). Mais, poursuivait-il, son choix s'inscrivait aussi dans le cadre de son engagement « pour la coexistence pacifique des deux cultures, celle de l'Ouest et celle de l'Est $»^{2}$ :

Je ressens personnellement profondément la contradiction entre les deux cultures: je suis fait de ces contradictions. Mes sympathies vont indéniablement au socialisme et à ce qu'on appelle le bloc de l'Est, mais je suis né et j'ai été élevé dans une famille bourgeoise et une culture bourgeoise $^{3}$. Cela me permet de collaborer avec ceux qui veulent approcher

\footnotetext{
1. Archives de la Fondation Nobel, Stockholm, 14 octobre 1964. Reproduit pour la première fois en fac-similé par Annie Cohen-Solal dans Sartre, p. 571.

2. Il convient de rappeler que la « coexistence pacifique » est une doctrine de politique extérieure qui fut théorisée par Staline en 1952, et reprise par Malenkov et Zhou En Lai en 1953 et surtout par Nikita Khrouchtchev lors du $\mathrm{XX}^{\mathrm{e}}$ Congrès du Parti communiste de l'Union soviétique. La déposition de ce dernier (le 15 octobre 1964, le Présidium du Soviet suprême d'URSS avait accepté sa « démission » comme Premier ministre) laissait planer un doute sur la politique à venir, et les suites données au « dégel». Quant aux « deux cultures» (bourgeoise et prolétarienne), le concept venait tout droit des Euvres complètes de Lénine, et avait eu comme conséquence la mise au pas de la science et des arts sous Jdanov et Lyssenko. Sartre connaissait bien ses classiques.

3. Ce qui était le thème des Mots.
} 
les deux cultures. J'espère cependant, bien entendu, que «le meilleur gagne ${ }^{1} \gg . C^{\prime}$ 'est-à-dire le socialisme.

C'est pourquoi je ne peux accepter aucune distinction distribuée par les hautes instances culturelles, pas plus à l'Ouest qu'à l'Ouest ${ }^{2}$, même si je comprends fort bien leur existence. Bien que toutes mes sympathies soient du côté socialiste, je serais donc bien incapable, tout aussi bien, d'accepter le prix Lénine, si quelqu'un voulait me le donner, ce qui n'est pas le cas.

Je sais bien que le prix Nobel en lui-même n'est pas un prix littéraire $d u$ bloc de l'Ouest, mais il est ce qu'on en fait, et il peut arriver des événements dont ne décident pas les membres de l'Académie suédoise.

C'est pourquoi, dans la situation actuelle, le prix Nobel se présente objectivement comme une distinction réservée aux écrivains de l'Ouest ou aux rebelles de l'Est ${ }^{3}$. On n'a pas couronné, par exemple, Neruda, qui est un des plus grands poètes sud-américains ${ }^{4}$. On n'a jamais parlé sérieusement de Louis Aragon, qui le mérite pourtant bien. Il est regrettable qu'on ait donné le prix à Pasternak avant de le donner à Cholokhov, et que la seule cuvre soviétique couronnée soit une œuvre éditée à l'étranger et interdite dans son pays. On aurait pu établir un équilibre par un geste semblable dans l'autre sens. Pendant la guerre d'Algérie, alors que nous avions signé la "Déclaration des 121 », j’aurais accepté le prix avec reconnaissance, parce qu'il n'aurait pas honoré que moi, mais aussi la liberté par laquelle nous luttions. Mais cela n'a pas eu lieu et ce n'est qu'après la fin des combats ${ }^{6}$ qu'on me décerne ce prix.

\footnotetext{
1. Khrouchtchev était persuadé qu'en vingt ans l'URSS aurait dépassé les États-Unis sur le plan économique.

2. Je souligne.

3. On appréciera l'expression. Du reste, l'appréciation de Sartre est fausse, puisque deux (très grands) auteurs considérés comme « staliniens », l'Islandais Halldór Laxness et l'Italien Salvatore Quasimodo, avaient reçu le prix Nobel - en 1956 et 1959, respectivement.

4. Pablo Neruda reçut le prix Nobel en 1971.

5. Boris Pasternak s'était vu décerner le prix Nobel en 1956. Il n'osa pas se rendre à Stockholm pour recevoir le prix, de peur d'être interdit de retour et, de fait, banni; il n'échappa du reste à ce sort que grâce à l'intervention de Nehru, admirateur de son œuvre. Mikhaïl Aleksandrovitch Cholokhov était un écrivain officiel du régime soviétique, qui lui décerna par deux fois le titre de « Héros du travail ». Il reçut le prix Nobel en 1965. On en vient finalement à penser que les seuls grands écrivains « de l'Ouest » à n'avoir pas eu le prix Nobel dans les années 1955-1970 pour des raisons politiques étaient de droite (Nabokov, Borges, Dos Passos, Malraux) ou compromis avec le fascisme (Malaparte, Morand).

6. C'est-à-dire l'indépendance de l'Algérie en 1962.
} 
Dans la motivation de l'Académie suédoise, on parle de liberté: c'est un mot qui invite à de nombreuses interprétations. À l'Ouest, on n'entend qu'une liberté générale: quant à moi, j'entends une liberté plus concrète qui consiste dans le droit d'avoir plus d'une paire de chaussures et de manger à sa faim. Il me paraît moins dangereux de décliner le prix que de l'accepter. Si je l'accepte, je me prête à ce que j'appellerais "une récupération objective ». Je considère [mon] "passé politique contro$v_{\text {versé }}^{1}$ » comme toujours valable, même si je suis prêt à reconnaître certaines erreurs passées au milieu de mes camarades ${ }^{2}$.

Sartre avait donc mis les points sur les « $\mathrm{i} »$ : Les Mots ne signifiait pas la fin de «l'engagement», et son attitude serait dictée au moins au partie par l'esprit de guerre froide - même s'il ne s'agit absolument pas pour moi de minimiser l'intérêt de sa réflexion sur le refus des honneurs et de la récupération.

Il en fallait plus pour décourager les mandarins de Cornell, comme le prouve la lettre de rappel que Desmorest prit l'initiative d'adresser à Jean-Paul Sartre le 12 novembre suivant:

Si vous vous souvenez lorsque vous avez eu la gentillesse de me faire savoir que vous viendriez à Cornell vous m'aviez également demandé de prier le Président Perkins de vous envoyer une lettre officielle à laquelle vous vouliez répondre personnellement. Cette lettre expédiée a dî être égarée. En voici donc un autre exemplaire.

Je tiens à vous exprimer encore une fois notre reconnaissance. Je vous rappelle que nous aurions également plaisir à recevoir Madame de Beauvoir si le cour lui en dit. D'autre part je fais le nécessaire en ce qui concerne votre billet d'avion et votre visa-le Consul des États-Unis à Paris m'a déjà fait savoir qu'il vous dispensera de la visite médicale obligatoire ainsi que des frais de visa.

Nous attendons donc avec une sympathique impatience votre réponse à l'invitation de Mr. Perkins, laquelle nous permettra immédiatement de prendre les mesures nécessaires, etc ${ }^{3}$.

\footnotetext{
1. Sartre avait été outré par un article du Figaro, le 14 octobre, insinuant que le prix Nobel allait lui être attribué en dépit de son engagement fréquent aux côtés des communistes: «On ne lui tiendra pas rigueur de son engagement politique controversé. »

2. Extraits du texte reproduit intégralement par Michel Contat, Pour Sartre, pp. 408-410.

3. Cornell University, Perkins Papers, \# 3-10-1022, box 6, folder 2.
} 
Desmorest est visiblement rendu nerveux par l'approche d'un cycle de conférences qui doit marquer le clou des célébrations pour le centenaire de l'université (1965). Il devait être rassuré, toutefois, par le fait que Sartre avait, via son secrétariat, pris un autre engagement aux États-Unis : une conférence au « Centre Y » à Manhattan, à l'invitation de la Young Men's and Young Women's Hebrew Association of New York, annoncée pour le 25 avril. Il voulait croire aussi que Sartre prendrait en compte les avancées sociales aux États-Unis, notamment sur la « question raciale »: Cornell prétendait donner l'exemple avec sa politique très volontariste en direction des « minorités », qui permit en effet à plus de 250 étudiants noirs américains et portoricains de s'inscrire en $1965^{1}$. L'administration regardait avec bienveillance de courageux étudiants, blancs et noirs, partir pour le Sud raciste dans les « Freedom buses ». Ils allaient manifester contre la ségrégation et le Ku Klux Klan, parfois au péril de leur vie: le 21 juin 1964 l'un d'entre eux, Michael Schwerner, fut assassiné dans la banlieue de Philadelphia, Mississipi, pour avoir fait inscrire des dizaines de Noirs sur les listes électorales.

De fait, Sartre fit confirmer sa venue à Cornell (par téléphone ou câble, fin février?), mais avec un sujet différent, " Histoire et éthique », qui semblait plus approprié à un large public: les 1000 tickets mis en vente pour l'événement prévu a Manhattan étaient partis comme des petits pains, et 2500 a 3000 personnes étaient attendues dans Bailey Hall, la plus grande salle de conférence de Cornell.

Cependant, l'Histoire « avec sa grande hache » (Perec) vint compliquer les choses. Le 31 janvier, les bombardiers américains quittèrent Okinawa pour le Sud-Vietnam (allié des Américains). Les premiers bombardements sur le Nord-Vietnam (tenu par les communistes) eurent lieu le 7 février avec une telle intensité et une telle violence destructrice que la campagne aérienne, qui devait durer trois ans, reçut le nom de « Rolling Thunder ». En outre, le 8 mars, eurent lieu les premiers déploiements de marines au sol. Toutefois, le vendredi 12 mars, la compagnie aérienne Trans-World Airlines informa Cornell que Sartre arriverait à Ithaca le

\footnotetext{
1. Dans le même temps, les militants de gauche dénonçaient le fait que Perkins siégeait au conseil d'administration de la Chase Manhattan Bank, qui réalisait d'énormes bénéfices en Afrique du Sud, malgré la politique d'apartheid que Sartre dénonçait sans relâche.
} 
2 avril (au lieu du 5). C'est seulement le 17 mars $^{1}$ que le philosophe fit envoyer un message lacunaire annulant sa venue: "The politics of violence practiced in Vietnam by the United States Government with the approval of the majority of the American people constitutes for me a major obstacle to my coming to the United States. " Immédiatement contacté par le New York Times, Sartre expliqua qu'un sondage réalisé par Gallup avait emporté sa décision : il montrait que moins de $20 \%$ des Américains voyaient dans l'engagement américain au Vietnam « une erreur " (a mistake) ; dès lors, estimait Sartre, " lorsqu'il y a une telle divergence d'opinions, le dialogue n'est pas possible ${ }^{2} »$.

Que ne lui expliqua-t-on que l'opposition à la guerre existait déjà, et qu'elle était forte sur un campus libéral comme Cornell? Il est vrai qu'elle tarda à se manifester publiquement. Rappelons que les premiers rassemblements connus sous le nom de teach-in (grands débats interactifs et contestataires associant professeurs et élèves) eurent lieu peu après le « coup » de Sartre, les 25 et 25 mars à l'université du Michigan (2500 personnes selon le New York Times, 25 mars 1965), et les 21 et 22 mai à Berkeley (30 000 personnes selon le même journal, 23 mai 1955). La même année, à Cornell, des manifestants firent scandale en organisant un des premiers sit-in anti-guerre, pendant un défilé militaire à Barton Hall.

Cette chronologie serrée rend difficile de porter un jugement sur la décision de Sartre, qui fut largement incomprise à Cornell, et continue de l'être. Les minutes de réunion du Messenger Lectures Committee donnent à penser que des mandarins comme Desmorest ou l'historien de la Grèce antique et futur néo-conservateur Donald Kagan se firent une raison. Ils cherchaient avant tout « de grands noms » ou des «stars $»^{3}$, puisque, trois mois après le « désastre Sartre » (« the Sartre Spring Disaster »), ils envisageaient d'inviter Malraux (!) ou Claude Lévi-

\footnotetext{
1. Sartre était coutumier des décisions de dernière minute - voir son appel à voter pour François Mitterrand en 1965.

2. «Sartre Cancels Lectures in U. S. Over American Role in Vietnam», New York Times, 18 mars 1965. Ce chiffre devait monter à $24 \%$ en août 1965, $35 \%$ en septembre 1966, $45 \%$ en décembre 1967 et $54 \%$ en août 1968 , et culminer a $60 \%$ en janvier 1973 (http://www.usatoday.com/news/polls/200511-15-iraq-poll.htm).

3. Anne Berger a souligné la force du paradigme hollywoodien dans le monde universitaire américain: " Il faudrait faire une analyse culturelle du succès de la notion de "succès" ", tous ceux qui connaissent les États-Unis l'ont remarqué, l'université américaine ne diffère pas fondamentalement de Hollywood. » (Labyrinthe, 24, 2006, p. 24).
} 
Strauss - vinrent finalement André Chastel (en 1966) et Jacqueline de Romilly (en 1967) ${ }^{1} \ldots$

Pour ce qui est des militants contre la sale guerre au Vietnam, il est probable que le refus de Sartre de donner des conférences à Cornell fut pour eux un encouragement. À tout le moins, n'importe quel professeur ou étudiant progressiste pouvait alors et devrait pouvoir aujourd'hui admettre le point de vue du Français : « les États-Unis évolueront [mieux] si on leur résiste que si on les encense ${ }^{2}$. »

Qu'en est-il vraiment? De récentes prises de position témoignent d'une blessure durable. Le 30 mai 2004, le juriste Jeffrey S. Lehman ${ }^{3}$, nouveau président de Cornell, dénonça vivement l'attitude de Sartre, lors de son discours de clôture de l'année universitaire et de remise des diplômes avec toges et chapeaux noirs carrés (une autre scène que les Français connaissent depuis l'enfance par les films et les séries télévisées). Il eut recours pour cela à... la métaphore sartrienne des mains sales:

Sartre a fait une erreur. En ne mettant pas les pieds aux États-Unis, un pays à ses yeux militariste, il a cru préserver sa pureté. En fait, il a perdu une occasion de parler, de débattre, de réfléchir-sur les liens entre éthique et histoire, et même sur cette guerre contre laquelle il prenait position. Alors que les circonstances faisaient une obligation morale de s'engager et de prendre parti, Sartre a fait le choix du désengagement pour ne pas se salir les mains ${ }^{4}$.

On peut se demander si, au-delà du dépit et du très mauvais procès ${ }^{5}$, ce genre de réaction ne traduit pas une double incompréhension culturelle : en France, l'intellectuel a traditionnellement le choix entre différents modes et champs d'action; non seulement l'université ne constitue pas son principal territoire, mais il ne devient intellectuel public que lorsque précisément il sort de la tour d'ivoire - où ses adversaires voudraient le

\footnotetext{
1. Archives encore non classées, Cornell University. Je remercie Elaine Engst, University Archivist, d'avoir porté ce document à ma connaissance.

2. Cité par Annie Cohen-Solal, «Sartre, référence obligée ou mauvais maître? », Le Monde, 20 juin 2006.

3. Lehman est un professeur de droit né en 1956. Diplômé de Cornell, il y avait notamment suivi les cours d'histoire et de culture françaises de Steve Kaplan. Il est depuis 2008 chancelier et doyen de la School of Transnational Law de l'Université de Pékin.

4. http://www.news.cornell.edu/campus/commencement04/Lehman.04.commencement.pdf. Je traduis.

5. Il est tout de même inadmissible d'accuser Sartre de désengagement par rapport au Vietnam, vu son implication dans le Tribunal Russell en 1966 et 1967.
} 
faire rentrer. Il en va autrement aux États-Unis où, comme le dit Régis Debray, « la Maison Blanche n'a rien à craindre de Harvard... le jus de cerveau, il y a des campus pour cela, et des sites Internet ${ }^{1} »$. Tout le monde n'accepte pas cette situation comme une fatalité, et il faut noter que certains activistes de gauche se réclament justement de Sartre: ainsi, le très médiatique Cornel West (Princeton University), présenté sur son propre site Internet comme « one of America's most provocative public intellectuals ${ }^{2} »$.

Deuxièmement, de nombreux Américains, même et surtout quand ils sont cultivés, ont du mal à admettre que la politique n'est pas forcément l'art du compromis ou la recherche du consensus rationnel. Sartre manifestait une intransigeance révolutionnaire, so what? Pensons à son engagement au côté des victimes de l'injustice et des violences d'État, contre la ségrégation raciale aux États-Unis ou en Afrique du Sud, ou lors du Tribunal Russell (ou Sartre-Russell) contre les crimes de guerre américains au Vietnam. Convenait-il, alors, de transiger?

Par conséquent, si l'on peut ressentir beaucoup d'amertume devant ses errances et ses aveuglements face aux « démocraties populaires $»^{3}$, on peut aussi être nostalgique de la liberté d'écrivain qu'il ne cessa d'affirmer vis-à-vis des pouvoirs et des grandeurs d'établissement.

Laurent Ferri

\footnotetext{
1. Régis Debray, I. f., suite et fin, Paris, Gallimard, 2000, p. 148. La démonstration est, comme toujours, impressionnante, mais Debray est-il bien sûr d'avoir plus d'influence en France que West ou Chomsky n'en ont aux États-Unis?

2. http://www.cornelwest.com.

3. Le pire était à venir, avec son engouement pour la tyrannie maoïste.
} 